\title{
Efficacy, effectiveness, efficiency
}

\author{
John Marley, Professor, Department of General Practice, University of \\ Adelaide, Adelaide
}

Index words: drug utilisation, cost-effectiveness, drug evaluation.

(Aust Prescr 2000;23:114-5)

How is it, that guidelines for treatment often seem unrelated to the patient sitting in front of the doctor? Guidelines are mostly based on evidence gathered from randomised controlled trials. These trials are very good at assessing efficacy - that is, can a treatment work? Despite this, trials are not without substantial biases. Many people may be screened before a few are chosen to be included in a study, yet the results of the study will be applied to the very people who were excluded. The population studied in trials tends to be young, male, white, suffering from a single condition and using a single treatment. Most patients, at least in general practice, do not fit this description. They often have multiple illnesses, take multiple medications and are either too young or too old to have been included in clinical trials. Perhaps we should accept a proposal to define efficacy in relation to medications as 'the extent to which a drug has the ability to bring about its intended effect under ideal circumstances, such as in a randomised clinical trial'.*

\section{In this issue...}

The new drugs reviewed in this issue have all been assessed for safety and efficacy. Although a treatment may be efficacious, John Marley points out that it may not be effective or efficient.

Heart failure needs effective treatment, but there are often difficulties in managing the condition. Henry Krum suggests some solutions to these therapeutic dilemmas. Peter Fletcher believes that beta blockers are the solution for some patients, even though these drugs were once contraindicated in heart failure.

While the cost-effectiveness of bisphosphonates may be questioned, they do have a role in some patients with low bone density, particularly postmenopausal women. John Martin and Vivian Grill inform us how the drugs work, while Peter Ebeling discusses their clinical use in osteoporosis.

The most effective treatment may not be a drug. In his article on panic disorder John Tiller tells us that cognitive behaviour therapy helps many patients. One of these patients is actor Garry McDonald who reveals how he overcame his anxiety.
Efficacy is not the same as effectiveness. ${ }^{1}$ A treatment is effective if it works in real life in non-ideal circumstances. In real life, medications will be used in doses and frequencies never studied and in patient groups never assessed in the trials. Drugs will be used in combination with other medications that have not been tested for interactions, and by people other than the patient - the 'over the garden fence' syndrome. Effectiveness cannot be measured in controlled trials, because the act of inclusion into a study is a distortion of usual practice. Effectiveness can be defined as 'the extent to which a drug achieves its intended effect in the usual clinical setting'.* It can be evaluated through observational studies of real practice. This allows practice to be assessed in qualitative as well as quantitative terms. ${ }^{2}$

Australia is well suited to conduct observational studies because we have a high standard of relatively unrestricted practice and good national databases, such as those held by the Health Insurance Commission. These databases can be used for validating researchers' separate database effectiveness studies. In America there are very large patient databases held by the Health Maintenance Organisations. Their size is impressive, but size is not everything. The data may have been collected primarily for billing and they may be incomplete. Clinical practice is often governed by protocols, and medications are limited to those supplied by the current preferred providers. The reimbursement mechanism for doctors may mean that they code conditions at the highest severity level. Patients belonging to one of these organisations may not represent the American population as a whole. In Britain, the General Practice Research Database, compiled from practice electronic records, is very useful, especially for studies in pharmacoepidemiology. The British enjoy relatively unrestricted clinical practice, but they do not have readily usable national datasets against which to check the validity of their database studies.

It is an irony that drugs are licensed for use almost exclusively on the results of controlled trials, yet they are withdrawn from use because of observational data that would not be acceptable to licensing authorities. Biases are present in observational studies, just as they are in trials, but they can be defined and often controlled for, giving these studies a much greater value than that currently awarded to them.

* From a suggested dictionary of pharmacoepidemiology by C. Ineke Neutel, University of Ottawa Institute on Health of the Elderly, Research Department, SCO Health Services. 43 Bruyere Street, Ottawa CANADA K1N 5 C8. 
Efficiency depends on whether a drug is worth its cost to individuals or society. The most efficacious treatment, based on the best evidence, may not be the most cost-effective option. It may not be acceptable to patients. In every country, rationing of health care is a reality. There is no country, however wealthy, that can afford to deliver all the health care possible to the whole of its population at all times. Rationing may be implicit or explicit, but it will happen. Good effectiveness and efficiency studies will make this rationing more informed.

Good practical guidelines, such as the Therapeutic Guidelines series, are clearly very important and extremely useful. They could be made even more relevant to the patient in front of the doctor, by being less dependent on efficacy studies. We should make more use of effectiveness and efficiency studies and abandon the censorship of the evidence drawn from them.

\author{
REFERENCES \\ 1. Haynes B. Can it work? Does it work? Is it worth it? Br Med J 1999;319: \\ 652-3. \\ 2. Greenhalgh T. Is my practice evidence-based? Br Med J 1996;313:957-8.
}

E-mail: john.marley@medicine.adelaide.edu.au

\section{Letters}

Letters, which may not necessarily be published in full, should be restricted to not more than 250 words. When relevant, comment on the letter is sought from the author. Due to production schedules, it is normally not possible to publish letters received in response to material appearing in a particular issue earlier than the second or third subsequent issue.

\section{Prescribing by numbers}

Editor, - It was interesting to see an article on the number needed to treat (NNT) (Aust Prescr 2000;23:38). NNT is better than looking at relative risk reductions but NNT still does not always give you a feel for the relevance of an intervention.

I believe clinical decision-making needs to consider two numbers. These are the paired absolute incidences.

$\mathrm{X}=$ Event rate control (the outcome with placebo, or the outcome if you do nothing)

$\mathrm{Y}=$ Event rate active (the outcome with treatment)

Consider a room full of 100 people with a clinical problem. Put it to them, 'Do nothing and the event will happen to $\mathrm{X}$ of you, and if all of you take the pill it will happen to Y of you.' Using the Helsinki Heart study as quoted in the article, how would 100 men respond if told 'Take gemfibrozil for five years and 4.1 of you will have an event, do nothing and 2.7 of you will have an event'? I suspect many would say why bother with treatment, but some would say OK.

Clinical decision-making needs to be made in the context of real people. Other comorbidity, patient attitude, patient expectations, the psychological burden of disease label, adverse effects, secondary costs (for example, more visits to the doctor) all need consideration. I believe that by looking at the two numbers ( $\mathrm{X}$ and $\mathrm{Y}$ ) I can get a better feel for the relevance of an intervention, and also inform my patients about 'doing something' versus 'doing nothing'.

I believe the treatment of risk and risk factors is greatly overrated, and that many are treated for risk without a genuine consideration of how much of a difference it could make for the individual. As the surgeons learn to withhold the knife, I believe we should learn to hold back the drug treatment of risk factors, not because there is no evidence, but because in the bigger picture it is irrelevant to the patient this will be facilitated by looking at the $\mathrm{X}$ and $\mathrm{Y}$ numbers.

Paul Neeskens

General Practitioner

Hervey Bay, Qld

\section{Medicines and the media}

Editor, - The Australian Prescriber editorial (Aust Prescr 2000;23:70-1) regarding reporting of medicines in the media is timely. On 13 April 2000, an article in the Adelaide 'Advertiser' included the headline 'Accepted safe levels of cholesterol "still too high", and pictured a young woman having a cholesterol test. The commentary continued, 'Worldwide evidence proved "normal" cholesterol levels in healthy men and women were too high, an international authority on heart disease said in Adelaide yesterday'. The article went on to talk about '...a new ultra-low dose cholesterol-reducing drug called cerivastatin, ...recently approved for use in Australia...'

Assuming a new study had been released assessing health outcomes associated with cerivastatin, we contacted the reporter. He could not provide any information to support the story, but suggested we contact the Adelaide marketing company publicising the visit of the overseas specialist. The marketing company supplied their media release, but could not provide a reference. They reported the media release was redrafted from one produced by a Sydney company. The Sydney marketing company also could not provide a reference. They said their media release was based on information supplied by Bayer, but they had returned all material to Bayer.

We rang Bayer on five occasions. The product manager was never available to speak to us, nor has he returned our call. The Adelaide marketing company, however, was more sympathetic. They rang us back to say the West of Scotland Coronary Prevention Study, a 1995 study involving pravastatin, was the basis for the story. Was the story 'news' or advertising? How can consumers tell the difference?

Libby Roughead and

Andrew Gilbert

School of Pharmacy and Medical Sciences

University of South Australia

Adelaide 